\title{
The Characteristic Function Method and Its Application to (1 + 1)-Dimensional Dispersive Long Wave Equation
}

\author{
Medhat M. Helal, Mohammad L. Mekky, Emad A. Mohamed \\ Department of Engineering Physics and Mathematics, Faculty of Engineering, Zagazig University, Zagazig, Egypt \\ Email: emadabdelhafiez@yahoo.com
}

Received October 19, 2011; revised November 24, 2011; accepted December 5, 2011

\begin{abstract}
In this paper, the characteristic function method is applied to seek traveling wave solutions of nonlinear partial differential equations in a unified way. We consider the Wu-Zhang equation (which describes $(1+1)$-dimensional dispersive long wave). The equations governing the wave propagation consist of a pair of non linear partial differential equations. The characteristic function method reduces the system of nonlinear partial differential equations to a system of nonlinear ordinary differential equations which is solved via the shooting method, coupled with Runge-kutta scheme. The results include kink-profile solitary wave solutions, periodic wave solutions and rational solutions. As an illustrative example, the properties of some soliton solutions for Wu-Zhang equation are shown by some figures.
\end{abstract}

Keywords: Characteristic Function Method; Wu-Zhang Equation

\section{Introduction}

Exact traveling wave solutions of nonlinear partial differential equations (NLPDEs) have been of a major concern for both mathematicians and physicists. Many efforts have been made on the study of NLPDEs. Long wave in shallow water is a subject of broad interests and has a long colorful history. Physically, it has a rich variety of phenomenological manifestation, especially the existence of waves permanent in form and robust in maintaining their entities through mutual interaction and collision as well as the remarkable property of exhibiting recurrences of initial data when circumstances should prevail. These characteristics are due to the intimate interplay between the roles of nonlinearity and dispersion. Mathematically, it has been noted that validity of theoretical models critically epends on the domain of underlying key parameters which characterize the specific motions to be modelled.

In the past few decades, many significant methods have been presented such as Bäcklund transformation [1], Darboux transformation [2], the extended tanh-function method [3], and the F-expansion method [4], Lie group analysis [5-8], homogeneous balance method [9], Jacobi elliptic function method [10] etc.

In 1985 Seshadri et al. [11] put the base-stone of the characteristic function method which was after then applied to solve a variety of nonlinear boundary value problems.
Lastly, Abd-el-malek et al. [12,13] applied the characteristic function method to solve some types of nonlinear partial differential equations which proved to be a powerful tool to be used.

In this present work, we shall derive the solutions of $(1+1)$-dimensional dispersive long wave equation via the characteristic function method, which depends on the application of a one-parameter group transformation to the system of the partial differential equations. These transformations reduce the number of independent variables in the system of partial differential equations by one. Our aim is to make use of the characteristic function method to represent the system of non-linear partial differential equations in the form of a system of ordinary differential equations which will be solved numerically using the shooting method, coupled with Runge-Kutta scheme.

\section{Formulation of the Problem}

Mathematically, it has been noted that validity of theoretical models critically depends on the domain of underlying key parameters which characterize the specific motions to be modelled. In [14], $\mathrm{Wu}$ and Zhang derived three sets of model equations for modelling nonlinear and dispersive long gravity waves travelling in two horizontal directions on shallow waters of uniform depth. Their comparative study of these models is directed to explore the intrinsic properties in physical and mathe- 
matical terms that these models possess. Omitting the higher order terms, one of these equations, Wu-Zhang (WZ) equation [14], can be written as:

$$
\begin{gathered}
u_{t}+u u_{x}+v u_{y}+w_{x}=0 \\
v_{t}+u v_{x}+v v_{y}+w_{y}=0 \\
w_{t}+(u w)_{x}+(v w)_{y}+\frac{1}{3}\left(u_{x x x}+u_{x y y}+v_{x x y}+v_{y y y}\right)=0
\end{gathered}
$$

where $w$ is the elevation of the water wave, $u$ is the surface velocity of water along $x$-direction and $v$ is the surface velocity of water along $y$-direction. The system (1)(3) can be reduced to $(1+1)$-dimensional dispersive long wave equation [14].

$$
\begin{gathered}
v_{t}+v v_{x}+w_{x}=0 \\
w_{t}+(w v)_{x}+\frac{1}{3} v_{x x x}=0
\end{gathered}
$$

where $w$ is as previously defined, $v$ is now is the surface velocity of water along $x$-direction. The system (4) and (5) can be traced back to the works of Broer [15], Kaup [16], Martinez [17], Kupershmidt [18], etc. A good understanding of all solutions of Equations (4) and (5) is very helpful for coastal and civil engineers to apply the nonlinear water wave model in a harbor and coastal design. Therefore, finding more types of exact solutions of Equations (4) and (5) is of fundamental interest in fluid dynamics. There is an amount of papers devoted to these equations [19-21]. We will seek the solution of Equations (4) and (5) considering the following initial and boundary conditions:

$$
\begin{aligned}
& w(x, 0)=\alpha(x), \quad v(x, 0)=\beta(x) \\
& w(0, t)=0, \quad v(0, t)=0, \\
& v_{x}(0, t)=f_{0}(t), \quad v_{x x}(0, t)=f_{1}(t)
\end{aligned}
$$

where $\alpha(x)$ and $\beta(x)$ are functions of $x$ and $f_{0}(t)$ and $f_{1}(t)$ are functions of $t$ to be specified.

\subsection{The Group Systematic Formulation under the Following Transformations}

$$
\begin{aligned}
& q_{1}=v_{t}, q_{11}=v_{t t}, q_{111}=v_{t t t}, \\
& q_{2}=v_{x}, q_{22}=v_{x x}, q_{222}=v_{x x x} \\
& q_{12}=v_{t x}, q_{122}=v_{x x t}, q_{112}=v_{t t x} \\
& r_{1}=w_{t}, r_{11}=w_{t t}, r_{111}=w_{t t t}, \\
& r_{222}=w_{x x x}, r_{12}=w_{t x}, r_{122}=w_{x x t}
\end{aligned}
$$

Considering the infinitesimal transformations in $(t, x, v$, w) given by

$$
\begin{aligned}
\bar{t}= & t+\varepsilon A(t, x, v, w)+O\left(\varepsilon^{2}\right) \\
\bar{x}= & x+\varepsilon B(t, x, v, w)+O\left(\varepsilon^{2}\right) \\
\bar{v}= & v+\varepsilon M(t, x, v, w)+O\left(\varepsilon^{2}\right) \\
\bar{w}= & w+\varepsilon N(t, x, v, w)+O\left(\varepsilon^{2}\right) \\
\bar{q}_{i}= & q_{i}+\varepsilon Q_{i}\left(t, x, v, w, q_{1}, q_{11}, q_{111}, q_{2}, q_{22},\right. \\
& \left.q_{222}, q_{12}, q_{122}, q_{112}, r_{1}, r_{11}, \cdots, \cdots\right)+O\left(\varepsilon^{2}\right) \\
\bar{r}_{i}= & r_{i}+\varepsilon R_{i}\left(t, x, v, w, r_{1}, r_{11}, r_{111}, r_{2}, r_{22},\right. \\
& \left.r_{222}, r_{12}, r_{122}, r_{112}, q_{1}, q_{11}, \cdots, \cdots\right)+O\left(\varepsilon^{2}\right) \\
\bar{q}_{j i}= & q_{j i}+\varepsilon Q_{j i}\left(t, x, v, w, p_{1}, p_{11}, p_{111}, p_{2},\right. \\
& \left.p_{222}, p_{12}, p_{122}, p_{112}, q_{1}, q_{11}, \cdots, \cdots\right)+O\left(\varepsilon^{2}\right) \\
\bar{r}_{j i}= & r_{j i}+\varepsilon R_{j i}\left(t, x, v, w, r_{1}, r_{11}, r_{111}, r_{2}, r_{22},\right. \\
& \left.r_{222}, r_{12}, r_{122}, r_{112}, q_{1}, q_{11}, \cdots, \cdots\right)+O\left(\varepsilon^{2}\right) \\
\bar{q}_{k j i}= & q_{k j i}+\varepsilon Q_{k j i}\left(t, x, v, w, q_{1}, q_{11}, q_{111}, q_{2}, q_{22},\right. \\
& \left.q_{222}, q_{12}, q_{122}, q_{112}, r_{1}, r_{11}, \cdots, \cdots\right)+O\left(\varepsilon^{2}\right) \\
\bar{r}_{k j i}= & r_{k j i}+\varepsilon R_{k j i} \varepsilon Q_{i}\left(t, x, v, w, q_{1}, q_{11}, q_{111},\right. \\
& \left.q_{2}, q_{22}, q_{222}, q_{12}, q_{122}, \cdots, \cdots\right)+O\left(\varepsilon^{2}\right)
\end{aligned}
$$

where $i, j$ and $k$ stand for $t, x$ and $A, B, M, N$, $Q_{i}, Q_{j i}, Q_{k j i}, R_{i j i}, R_{i}, R_{j i}, R_{k j i}$ are the infinitesimals of the group of transformations. Hence Equations (4) and (5) becomes:

$$
\begin{aligned}
& G_{1}=q_{1}+v q_{2}+r_{2} \\
& G_{2}=r_{1}+v r_{2}+\frac{1}{3} r_{222}
\end{aligned}
$$

To calculate the prolongation of a given transformation, we need to differentiate Equations (9) and (10) with respect to each of the variables $t, x$. To do this we introduce the following total derivatives:

$$
\begin{aligned}
D t(f)= & \frac{\partial}{\partial t}(f)+\frac{\partial}{\partial t} w(t, x) \cdot \frac{\partial}{\partial w}(f) \\
& +\frac{\partial}{\partial t} w(t, x) \cdot \frac{\partial}{\partial w}(f)+\frac{\partial}{\partial t} v(t, x) \cdot \frac{\partial}{\partial v}(f) \\
& +\frac{\partial^{2}}{\partial t^{2}} w(t, x) \cdot \frac{\partial}{\partial w_{t}}(f)+\frac{\partial^{2}}{\partial t^{2}} v(t, x) \cdot \frac{\partial}{\partial v_{t}}(f) \\
& +\frac{\partial^{2}}{\partial t^{2}} w(t, x) \cdot \frac{\partial}{\partial w_{t}}(f)+\frac{\partial^{2}}{\partial t \partial x} w(t, x) \cdot \frac{\partial}{\partial w_{t}}(f) \\
& +\frac{\partial^{2}}{\partial t \partial x} v(t, x) \cdot \frac{\partial}{\partial v_{t}}(f)+\cdots+\cdots
\end{aligned}
$$

And 


$$
\begin{aligned}
D x(f)= & \frac{\partial}{\partial x}(f)+\frac{\partial}{\partial x} w(t, x) \cdot \frac{\partial}{\partial w}(f) \\
& +\frac{\partial}{\partial x} v(t, x) \cdot \frac{\partial}{\partial v}(f)+\frac{\partial}{\partial x} w(t, x) \cdot \frac{\partial}{\partial w}(f) \\
& +\frac{\partial^{2}}{\partial x^{2}} w(t, x) \cdot \frac{\partial}{\partial w_{x}}(f)+\frac{\partial^{2}}{\partial x^{2}} v(t, x) \cdot \frac{\partial}{\partial v_{x}}(f) \\
& +\frac{\partial^{2}}{\partial x^{2}} w(t, x) \cdot \frac{\partial}{\partial w_{x}}(f)+\frac{\partial^{2}}{\partial t \partial x} w(t, x) \cdot \frac{\partial}{\partial w_{x}}(f) \\
& +\frac{\partial^{2}}{\partial t \partial x} v(t, x) \cdot \frac{\partial}{\partial v_{x}}(f)+\cdots+\cdots
\end{aligned}
$$

where

$$
\begin{aligned}
& Q^{i}=D_{i}(M 2)-v_{t} D_{i}(A)-v_{x} D_{i}(B) \\
& R^{i}=D_{i}(M 3)-w_{t} D_{i}(A)-w_{x} D_{i}(B) \\
& Q^{j i}=D_{i}\left(Q^{j}\right)-v_{j t} D_{i}(A)-v_{j x} D_{i}(B) \\
& R^{j i}=D_{i}\left(R^{j}\right)-w_{j t} D_{i}(A)-w_{j x} D_{i}(B) \\
& Q^{k j i}=D_{i}\left(Q^{k j}\right)-v_{k j t} D_{i}(A)-v_{k j x} D_{i}(B) \\
& R^{k j i}=D_{i}\left(R^{k j}\right)-w_{k j t} D_{i}(A)-w_{k j x} D_{i}(B)
\end{aligned}
$$

where $i, j$ and $k$ stand for $x$ and $t$.

\subsection{The Invariance Analysis}

Under the infinitesimal group of transformation, the system of differential Equations (4) and (5) on the form $G_{i}=$ 0 , for $i=1$, 2, will be invariant if

$$
D G_{i}=0
$$

where $D$ is the total derivative defined by:

$$
\begin{aligned}
D_{i}= & \partial_{i}+u_{i} \partial u+v_{i} \partial v+u_{i x} \partial u_{x} \\
& +v_{i x} \partial v_{x}+u_{i t} \partial u_{t}+v_{i t} \partial v_{t}+\cdots
\end{aligned}
$$

The operator $D$ can be rewritten as:

$$
\begin{aligned}
D= & A \frac{\partial}{\partial t}+B \frac{\partial}{\partial x}+M \frac{\partial}{\partial v}+N \frac{\partial}{\partial w} \\
& +Q_{1} \frac{\partial}{\partial q_{1}}+Q_{2} \frac{\partial}{\partial q_{2}}+R_{1} \frac{\partial}{\partial r_{1}}+R_{2} \frac{\partial}{\partial r_{2}} \\
& +R_{11} \frac{\partial}{\partial r_{11}}+R_{22} \frac{\partial}{\partial r_{22}}+R_{12} \frac{\partial}{\partial r_{12}} \\
& +Q_{111} \frac{\partial}{\partial q_{111}}+Q_{222} \frac{\partial}{\partial q_{222}}+R_{111} \frac{\partial}{\partial r_{111}} \\
& +R_{222} \frac{\partial}{\partial r_{222}}+Q_{112} \frac{\partial}{\partial q_{112}}++R_{112} \frac{\partial}{\partial r_{112}} \\
& +Q_{122} \frac{\partial}{\partial q_{122}}++R_{122} \frac{\partial}{\partial r_{122}}+\cdots+\cdots
\end{aligned}
$$

Invariance of Equations (9) and (10) under Equation (16) gives:

$$
\begin{aligned}
D G_{1}= & Q_{1}+M \frac{\partial v}{\partial x}+v Q_{2}+R_{2}=0 \\
D G_{2}= & R_{1}+M \frac{\partial w}{\partial x}+v R_{2}+N \frac{\partial v}{\partial x} \\
& +w Q_{2}+\frac{1}{3} R_{222}=0
\end{aligned}
$$

We conclude that the system of Equations (4) and (5) will be invariant under an infinitesimal group of transformations. Therefore, it is now possible to reduce the number of independent variables by one.

The characteristic system of Equations (4) and (5) is given by:

$$
\frac{d x}{B_{1}+B_{2} t+B_{3} x}=\frac{d t}{A_{1}+3 B_{3} t}=\frac{d v}{B_{2}-2 B_{3} v}=\frac{d w}{-4 B_{3} w}
$$

where $A_{1}, B_{1}, B_{2}$ and $B_{3}$ are real constants.

\subsection{The Reduction to Ordinary Differential Equations}

Our aim is to make use of CFM to represent the problem in the form of ordinary differential equations. Then we have to proceed our analysis to complete the transformation in the next section in three Cases (1.1), (1.2) and Case (2) respectively.

\section{Experiments and Results}

In this section similarity transformation can be obtained by solving the characteristic system (19) which gives the similarity transformation for the dependent and independent variables.

\section{Case (1)}

In Equation (19), assuming $A_{1}=B_{1}=B_{2}=1.0$ and $B_{3}$ $=0$, then we get the following system:

$$
\frac{\mathrm{d} x}{B_{1}+B_{2} t}=\frac{\mathrm{d} t}{A_{1}}=\frac{\mathrm{d} v}{B_{2}}=\frac{\mathrm{d} w}{0}
$$

Solving these system leads to:

$$
\eta=x-t-\frac{1}{2} t^{2}
$$

where $\eta(x, t)$ is the similarity variable of the independent variables. And the similarity functions of the dependent variables are:

$$
\begin{gathered}
F_{1}(\eta)=v-t \\
F_{2}(\eta)=w
\end{gathered}
$$

where $F_{1}(\eta)$ refer to the surface velocity and $F_{2}(\eta)$ refer to the elevation of the water wave and they are arbitrary functions of $\eta$. Substituting the self-similar variables $v$ 
and $w$ from the Equations (22) and (23) into Equations (4) and (5) yields a system of ordinary differential equations in the similarity variable $\eta$ :

$$
\begin{aligned}
& F_{2}^{\prime}-F_{1}^{\prime}+F_{1} F_{1}^{\prime}+1=0 \\
& F_{2} F_{1}^{\prime}-F_{2}^{\prime}+F_{1} F_{2}^{\prime}+\frac{1}{3} F_{1}^{\prime \prime \prime}=0
\end{aligned}
$$

Equations (24) and (25) are solved numerically applying the shooting method coupled with Runge-kutta scheme using two groups of initial conditions illustrated in Cases (1.1) and (1.2) as follow.

Case (1.1)

Solving Equations (24) and (25), under the following boundary conditions:

$$
\begin{aligned}
& F_{1}(0)=0, F_{2}(0)=0 \\
& F^{\prime}(0)=-0.01, F_{1}^{\prime \prime}(0)=1.20
\end{aligned}
$$

The obtained results are presented diagrammatically in (Figure 1).
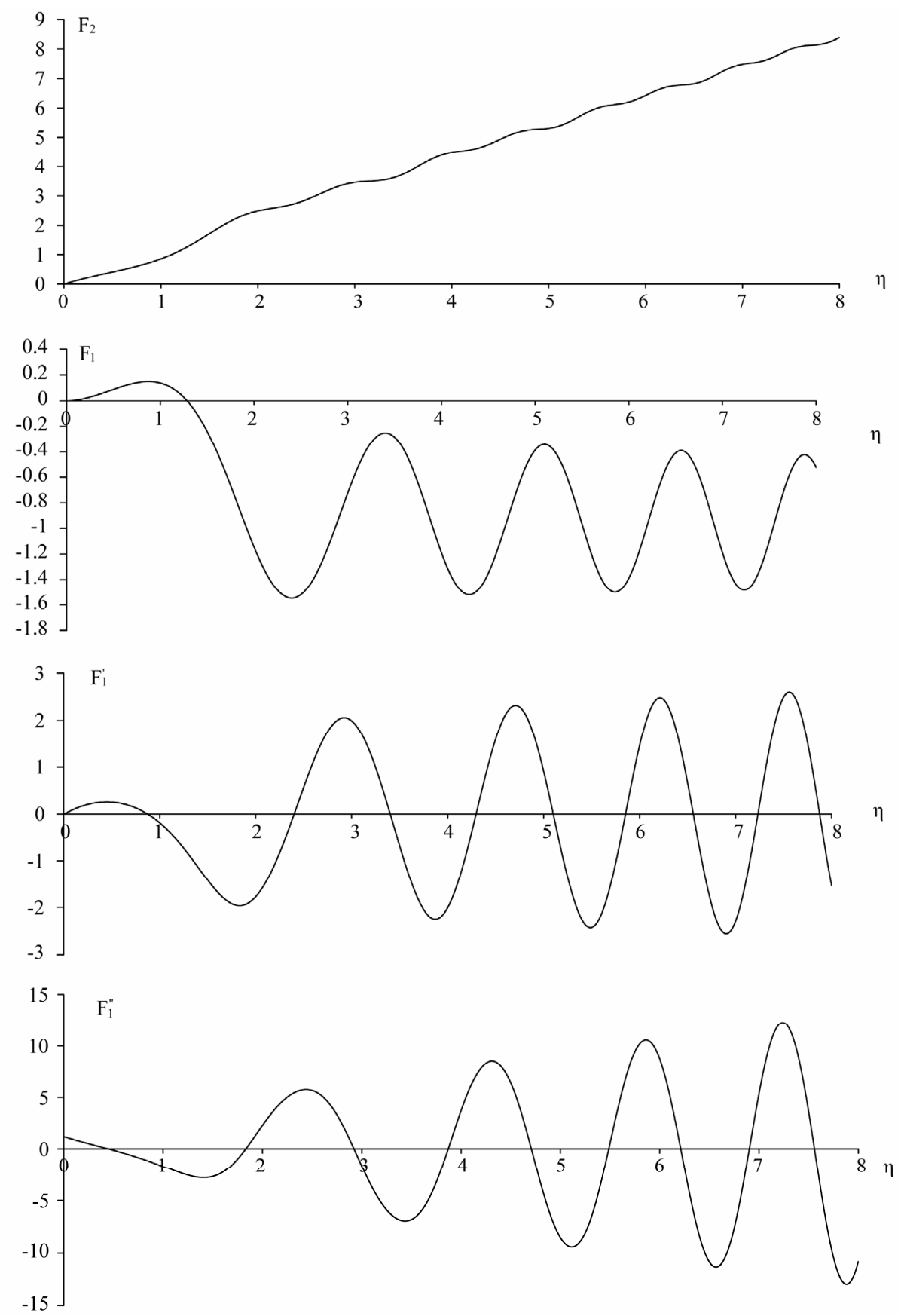

Figure 1. Solution of $(1+1)$-dimensional dispersive long wave Equations (24) and (25) at $A_{1}=B_{1}=B_{2}=1.0$. Where $F_{1}$ refer to the surface velocity and $F_{2}$ refer to the elevation of the water wave. 


\section{Case (1.2)}

Also we solve Equations (24) and (25) under the following boundary conditions:

$$
\begin{aligned}
& F_{1}(0)=0, F_{2}(0)=0 \\
& F^{\prime}(0)=-4, F_{1}^{\prime \prime}(0)=22
\end{aligned}
$$

In addition to, the boundary conditions which illustrated in the figures. The obtained results are presented diagrammatically in (Figure 2).

Case (2)

In Equation (19), assuming $B_{1}=B_{2}=B_{3}=0$ and $A_{1} \neq$ 0 , then we get the following system:
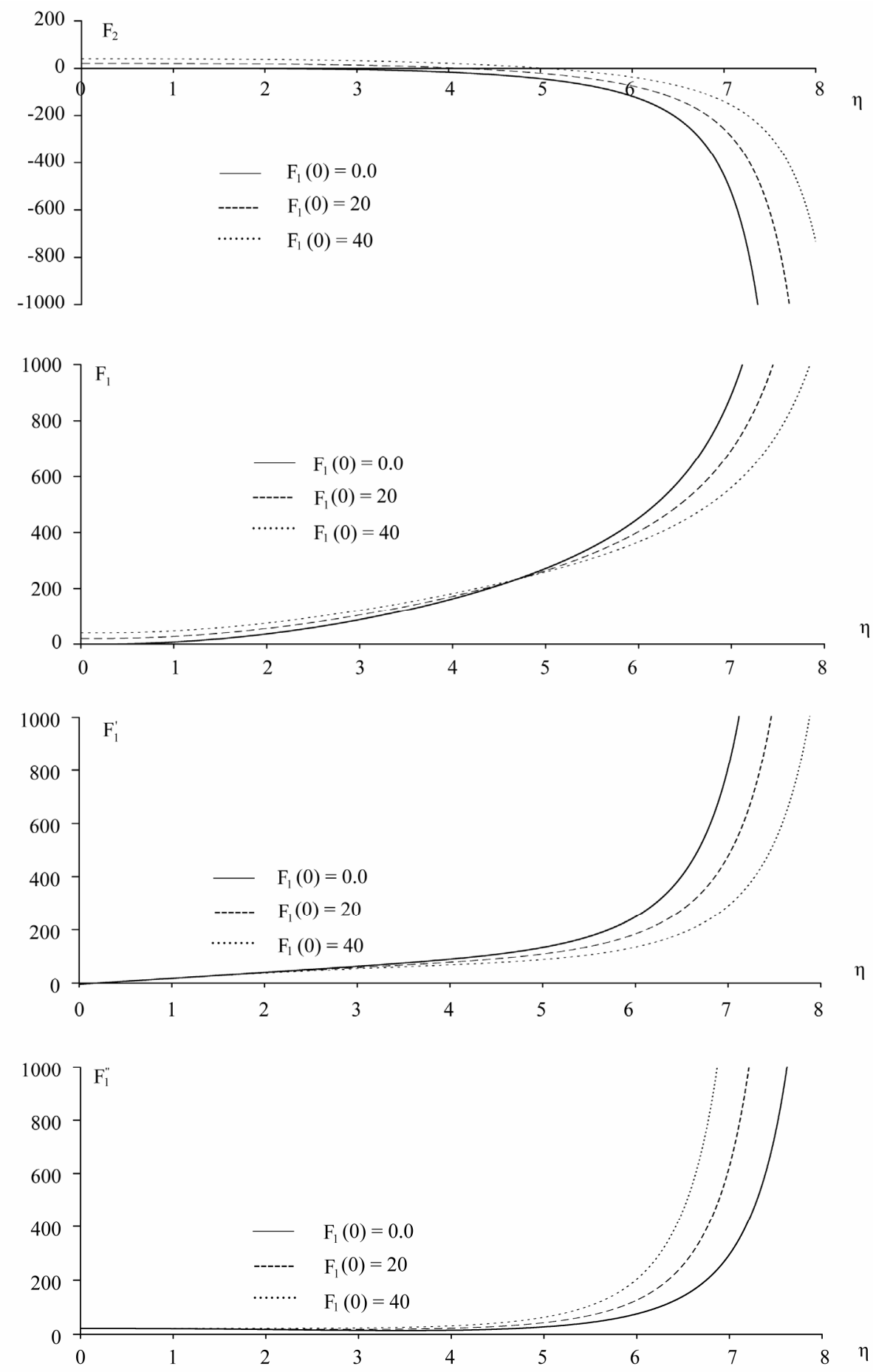

Figure 2. Solution of $(1+1)$-dimensional dispersive long wave Equations (24) and (25) at $A_{1}=B_{1}=B_{2}=1.0$. Where $F_{1}$ refer to the surface velocity and $F_{2}$ refer to the elevation of the water wave. 


$$
\frac{\mathrm{d} x}{0}=\frac{\mathrm{d} t}{A_{1}}=\frac{\mathrm{d} v}{0}=\frac{\mathrm{d} w}{0}
$$

In this case, similarity transformation is given as:

$$
\begin{gathered}
\eta=A_{1} x \\
F_{1}(\eta)=v \\
F_{2}(\eta)=w
\end{gathered}
$$

Substituting $F_{1}(\eta)$ and $F_{2}(\eta)$ from Equations (30), (31) into Equations (4) and (5) gives:

$$
F_{1} F_{1}^{\prime}+F_{2}^{\prime}=0
$$

$$
F_{2} F_{1}^{\prime}+F_{1} F_{2}^{\prime}+\frac{1}{3} A_{1}^{2} F_{1}^{\prime \prime \prime}=0
$$

The numerical solutions under the following boundary conditions:

$$
\begin{aligned}
& F_{1}(0)=0, F_{2}(0)=0 \\
& F_{1}^{\prime}(0)=-0.7, F_{1}^{\prime \prime}(0)=0.5
\end{aligned}
$$

In addition to, the boundary conditions which illustrated in the figures. The obtained results are presented diagrammatically in (Figure 3).
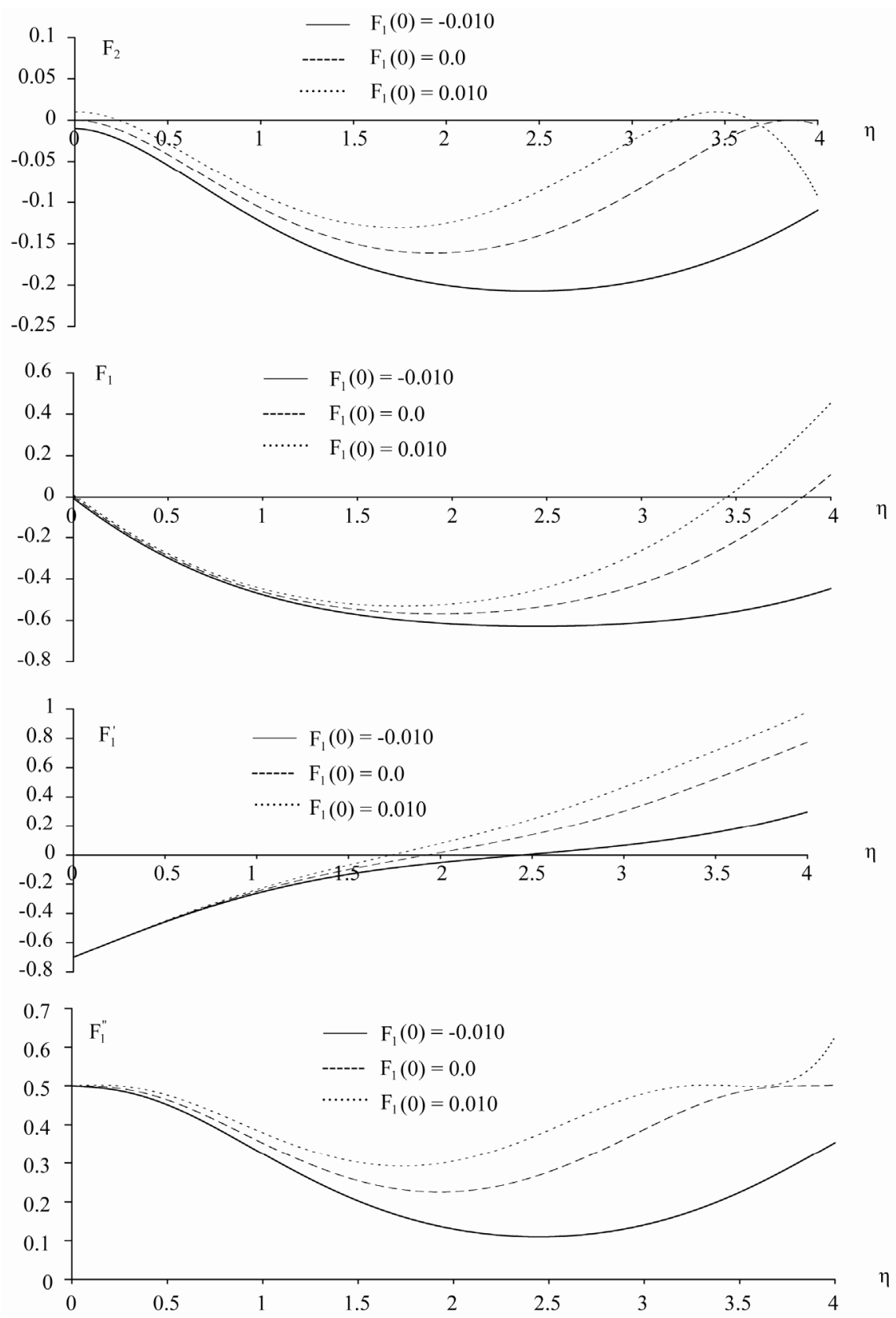

Figure 3. Solution of $(1+1)$-dimensional dispersive long wave Equations (32) and (33) at $A_{1}=1.0$. Where $F_{1}$ refer to the surface velocity and $F_{2}$ refer to the elevation of the water wave. 
Remark: Using MAPLE, we have verified all solutions we obtained by putting them back into the original NPDEs, WZ Equation (4) and (5). And it is worthy to point out that the new forms of solutions in Case (1), where the similarity variable $\eta=x-t-0.5 t^{2}$ cannot be obtained by the generalized extended rational expansion method [22], and has not reported in the literature.

\section{Conclusion}

In this work, we have used the characteristic function technique to obtain similarity reductions of nonlinear partial differential Equations (4) and (5), for (1 + 1)-dimensional dispersive long wave equation (DLWE) in shallow water with uniform depth. The resulting system of non-linear ordinary differential with the associated boundary conditions is solved numerically using shooting method, coupled with Runge-Kutta scheme. The numerical solution was carried out for various values of the parameters of the equations to study different cases and illustrate the effect of critical and special values on the performance of velocity and elevation of water wave.

\section{REFERENCES}

[1] M. Wadati, H. Sanuki and K. Konno, "Relationships among Inverse Method, Bäcklund Transformation and an Infinite Number of Conservation Laws," Progress of Theoretical Physics, Vol. 53, No. 2, 1975, pp. 419-436. doi:10.1143/PTP.53.419

[2] V. A. Matveev and M. A. Salle, "Darboux Transformations and Solitons,” Springer-Verlag, Berlin, Heidelberg, 1991.

[3] E. G. Fan, "Extended Tanh-Function Method and Its Applications to Nonlinear Equations," Physics Letters A, Vol. 277, No. 4-5, 2000, pp. 212-218. doi:10.1016/S0375-9601(00)00725-8

[4] Y. B. Zhou, M. L. Wang and Y. M. Wang, "Periodic Wave Solutions to a Coupled KdV Equations with Variable Coefficients," Physics Letters A, Vol. 308, No. 1, 2003, pp. 31-36.

[5] P. J. Olever, "Applications of Lie Groups to Differential Equations,” Springer, New York, 1968.

[6] G. W. Bluman and S. Kumei, "Symmetries and Differential Equations,” Springer, New York, 1989.

[7] H. Stephen,” Differential Equations: Their Solutions Using Symmetries,” Cambridge University Press, Cambridge, 1990. doi:10.1017/CBO9780511599941

[8] N. H. Ibragimov, "CRC Handbook of Lie Group Analysis of Differential Equation,” CRC Press, Boca Raton, 1996.

[9] M. L. Wang, Y. B. Zhou and Z. B. Li, "Application of a Homogeneous Balance Method to Exact Solutions of Nonlinear Equations in Mathematical Physics,” Physics Letters A, Vol. 216, No. 1-5, 1996, pp. 67-75.

\section{doi:10.1016/0375-9601(96)00283-6}

[10] E. G. Fan and J. Zhang,” Applications of Jacobi Elliptic Function Method to Special-Type Nonlinear Equations," Physics Letters A, Vol. 305, No. 6, 2002, pp.383-392. doi:10.1016/S0375-9601(02)01516-5

[11] R. Seshadri and T. Y. Na, "Group Invariance in Engineering Boundary Value Problems,” Springer-Verlag, New York, 1985. doi:10.1007/978-1-4612-5102-6

[12] M. B. Abd-el-Malek and M. M. Helal, "Characteristic Function Method for Classification of Equations of Hydrodynamics of a Perfect Luid," Journal of Computational and Applied Mathematics, Vol. 182, No. 1, 2005, pp. 105-116. doi:10.1016/j.cam.2004.11.042

[13] M. B. Abd-el-Malek and M. M. Helal, "The Characteristic Function Method and Exact Solutions of Nonlinear Sheared Flows with Free Surface under Gravity," Journal of Computational and Applied Mathematics, Vol. 189, No. 1-2, 2006, pp. 2-21. doi:10.1016/j.cam.2005.04.038

[14] T. Y. Wu and J. E. Zhang, “On Modeling Nonlinear Long Wave,” In: L. P. Cook, V. Roytbhurd and M. Tulin, Eds., Mathematics Is for Solving Problems, Society for Industrial and Applied Mathematics, Philadelphia, 1996, p. 233.

[15] L. J. F. Broer, “Approximate Equations for Long Water Waves,” Applied Scientific Research, Vol. 31, No. 5, 1975, pp. 377-395. doi:10.1007/BF00418048

[16] D. J. Kaup, "Finding Eigenvalue Problems for Solving Nonlinear Evolution Equations," Progress of Theoretical Physics, Vol. 54, No. 1, 1975, pp. 72-78. doi:10.1143/PTP.54.72

[17] L. Martinez, "Schrodinger Spectral Problems with EnergyDependent Potentials as Sources of Nonlinear Hamiltonian Evolution Equations,” Journal of Mathematical Physics, Vol. 21, No. 9, 1980, pp. 2342-2349. doi:10.1063/1.524690

[18] B. A. Kupershmidt, "Mathematics of Dispersive Water Waves," Communications in Mathematical Physics, Vol. 99, No. 1, 1985, pp. 51-73. doi:10.1007/BF01466593

[19] C. L. Chen and S. Y. Lou, "Soliton Excitations and Periodic Waves without Dispersion Relation in Shallow Water System,” Chaos, Solitons \& Fractals, Vol. 16, No. 1, 2003, pp. 27-35. doi:10.1016/S0960-0779(02)00148-0

[20] M. L. Wang, "Solitary Wave Solutions for Variant Boussinesq Equations,” Physics Letters A, Vol. 199, No. 3-4, 1995, pp. 169-172. doi:10.1016/0375-9601(95)00092-H

[21] X. D. Zheng, Y. Chen and H. Q. Zhang, "Generalized Extended Tanh-Function Method and Its Application to $(1+1)$ Dimensional Dispersive Long Wave Equation,” Physics Letters A, Vol. 311, No. 2-3, 2003, pp. 145-157. doi:10.1016/S0375-9601(03)00451-1

[22] X. Zeng, D. Wang and S. Wang, “A Generalized Extended Rational Expansion Method and Its Application to $(1+1)$ Dimensional Dispersive Long Wave Equation,” Applied Mathematics and Computation, Vol. 212, No. 2, 2009, pp. 296-304. doi:10.1016/j.amc.2009.02.020 